\title{
Neuroradiological Evolution of Glycaemic Hemichorea-Hemiballism and the Possible Role of Brain Hypoperfusion
}

\author{
Lucio Marinelli ${ }^{1,2}$, Davide Maggi ${ }^{3,4}$, Carlo Trompetto ${ }^{1,2}$, Paolo Renzetti ${ }^{5}$ \\ ${ }^{1}$ Department of Neuroscience, Rehabilitation, Ophthalmology, Genetics, Maternal and Child Health, University of Genoa, Italy \\ ${ }^{2}$ Department of Neuroscience, IRCCS Ospedale Policlinico San Martino, Genoa, Italy \\ ${ }^{3}$ Diabetology Clinic, Department of Internal Medicine, University of Genoa, Italy \\ ${ }^{4}$ Diabetology Clinic, IRCCS Ospedale Policlinico San Martino, Genoa, Italy \\ ${ }^{5}$ Department of Neuroradiology, IRCCS Ospedale Policlinico San Martino, Genoa, Italy
}

\section{Doi: 10.12890/2019_001257 - European Journal of Case Reports in Internal Medicine - C EFIM 2019}

Received: 21/08/2019

Accepted: 20/09/2019

Published: $23 / 10 / 2019$

How to cite this article: Marinelli L, Maggi D, Trompetto C, Renzetti P. Neuroradiological evolution of glycaemic hemichorea-hemiballism and the possible role of brain hypoerfusion. EJCRIM 2019;6: doi:10.12890/2019_001257.

Conflicts of Interests: The Authors declare that there are no competing interest

This article is licensed under a Commons Attribution Non-Commercial 4.0 License

\section{ABSTRACT}

Background: Lateralized involuntary movements consistent with hemichorea-hemiballism (HCHB) may appear following the development of contralateral haemorrhagic or ischaemic lesions of the basal ganglia, particularly the striatum (caudate nucleus and putamen). This condition is called vascular HCHB, but the same symptoms can be caused by a completely different striatal lesion. Glycaemic HCHB may occur in patients with uncontrolled hyperglycaemia: basal ganglia hyperdensity is seen on brain CT, while increased T1 signal intensity and reduced susceptibility-weighted imaging (SWI) and gradient-echo sequences (T2*-GRE) are detected on MRI.

Case description: An 83-year-old man with multiple vascular risk factors and uncontrolled chronic hyperglycaemia was admitted for ischaemic stroke presenting with dysarthria and mild left hemiparesis. No involuntary movements were reported at admission. The emergent brain CT scan was negative for vascular acute lesions, while a mild bilateral hyperdensity of the striata was detectable. Involuntary movements on the left side of the body, consistent with $\mathrm{HCHB}$, appeared 27 days later. The alterations on brain CT completely disappeared after 3 months. On brain MRI, the T1 signal alterations resolved after 10 months, while SWI and T2*-GRE sequences showed persisting alterations after 2 years. Discussion: Detailed brain imaging demonstrated evolution of striatal alterations of glycaemic HCHB before the appearance of involuntary movements and during the following 2 years. The association between ischaemic stroke and glycaemic HCHB favours the hypothesis that chronic hyperglycaemia more likely determines striatal alterations and the clinical picture of HCHB when vascular hypoperfusion also occurs.

\section{LEARNING POINTS}

- Hemichorea-hemiballism (HCHB) may appear in patients with uncontrolled hyperglycaemia accompanied by typical neuroradiological features consisting of striatal alterations detectable on brain CT and MRI, often bilaterally.

- Unusually, striatal alterations can be detected before the appearance of involuntary movements and are fully reversible on brain CT and T1-MRI sequences.

- Brain hypoperfusion may facilitate the appearance of typical HCHB in patients with chronic hyperglycaemia.

\section{KEYWORDS}

Hemichorea, hemiballism, athetosis, hyperglycemia, hypoperfusion 


\section{INTRODUCTION}

The most frequent causes of sporadic chorea are vascular lesions of the basal ganglia; in particular, unilateral lesions of the basal ganglia may determine contralateral involuntary movement ${ }^{[1]}$. In hemichorea, lateralized movements are rapid, irregular and affect the distal parts of the limbs, while in hemiballism movements are proximal and flinging. Both types of involuntary movements often coexist in a condition called hemichorea-hemiballism (HCHB).

Patients with stroke sometimes develop HCHB along with hemiparesis; in the majority of cases, involuntary movements improve only partially over time. Patients with uncontrolled hyperglycaemia may develop HCHB without evidence of vascular lesions in a condition called glycaemic $\mathrm{HCHB}^{[2]}$. In vascular hemichorea, a contralateral ischaemic or haemorrhagic lesion of the basal ganglia can be demonstrated, while neuroradiological findings are completely different in patients with glycaemic HCHB. Brain CT shows hyperdensity, while brain magnetic resonance imaging (MRI) shows T1-weighted hyperintensity usually involving the striatum contralateral to the affected side or bilaterally. Susceptibility weighted imaging (SWI) and gradient echo sequence T2*-weighted images (T2*-GRE) usually show signal reduction and negative DWI and ADC signals in the same region ${ }^{[3-6]}$. There is no mass effect and the lesions may be fully reversible $\mathrm{e}^{[7]}$. The pathological mechanisms of such lesions are still elusive: some authors suggest petechial haemorrhage, metal deposition (e.g., calcium, manganese) or mild ischaemia causing astrocyte swelling ${ }^{[8]}$. The temporal evolution of the neuroimaging has not been widely studied and only one report has described typical findings on brain CT before symptom onset ${ }^{[9]}$.

\section{CASE DESCRIPTION}

We describe the case of an 83-year-old man with a history of arterial hypertension, mild chronic renal insufficiency, and prostate cancer treated with radiation therapy, bicalutamide, tamoxifen and triptorelin. He had had a previous mild ischaemic stroke with transient right facio-brachio-crural hemiparesis followed by pneumonia caused by Staphylococcus aureus with complete recovery. At that time, blood glucose levels were 114 and $99 \mathrm{mg} / \mathrm{dl}$ (normal values $65-110 \mathrm{mg} / \mathrm{dl}$ ). After 6 years he presented with thrombosis of the right common iliac vein treated with low-molecular-weight heparin, closely followed by myocardial infarction, consequent coronary stent implantation and double antiplatelet therapy (dipyridamole $200 \mathrm{mg} /$ day plus acetylsalicylic acid $150 \mathrm{mg} /$ day).

Four months later, worsening of walking ability was closely followed by dysarthria about 1 week later. The patient was admitted to our hospital where he was diagnosed with mild ischaemic stroke, showing mild left hemiparesis without sensory impairment. An emergent brain CT was negative for acute lesions and only former small chronic ischaemic lesions were reported (Fig. 1,-26 days). Later review showed mild bilateral hypodensity of the striata was already present, which became clearer on another CT scan performed 2 days later (Fig. 1, -24 days). No involuntary movements were reported at admission or during the following days while the patient was in the university neurology ward. Blood tests showed very high blood glucose (505 mg/dl) and glycated haemoglobin of $15.7 \%$ (normal values 4.3-5.8\%). Subcutaneous insulin therapy was started as suggested by the consultant diabetologist. Mild dysphagia was present but no parenteral nutrition was necessary. Twenty-seven days after admission, while the patient was in the neurorehabilitation ward, he developed involuntary movements on the left side of his body, consistent with moderate HCHB. He was diagnosed with glycaemic HCHB and started treatment with olanzapine (up to $5 \mathrm{mg} / \mathrm{day}$ for 7 days), then haloperidol (up to $3 \mathrm{mg} /$ day for 20 days) and finally tetrabenazine (up to $25 \mathrm{mg} / \mathrm{day}$ for about 6 months), with a partial reduction in involuntary movements.

The first brain MRI was performed 7 days after HCHB onset and confirmed an increase in signal intensity on axial T1-weighted imaging in both striata (Fig. 2). Notably, the hyperintensity was asymmetrical since the right striatum, contralateral to the affected side of the body, was clearly more hyperintense than the left striatum; DWI and ADC were negative. Such asymmetry was much less evident on CT scans. The patient underwent neurological follow-up which showed a gradual reduction in HCHB. Tetrabenazine was stopped and involuntary movement reappeared only while the patient was speaking (Video 1). This condition remained unaltered during 26 months of follow-up. To monitor how lesions evolved, brain CT and MRI were repeated for up to 1.5 years after HCHB onset. Striatal hyperdensity on CT scans was no longer detectable after about 3 months (Fig. 1). Striatal hyperintensity on T1 MRI disappeared completely after 10 months, while striatal hypointensity on SWI and T2*-GRE still persisted 1.5 years later (Fig. 2). Table 1 shows the times and results of blood glucose and glycated haemoglobin tests and brain neuroimaging. 

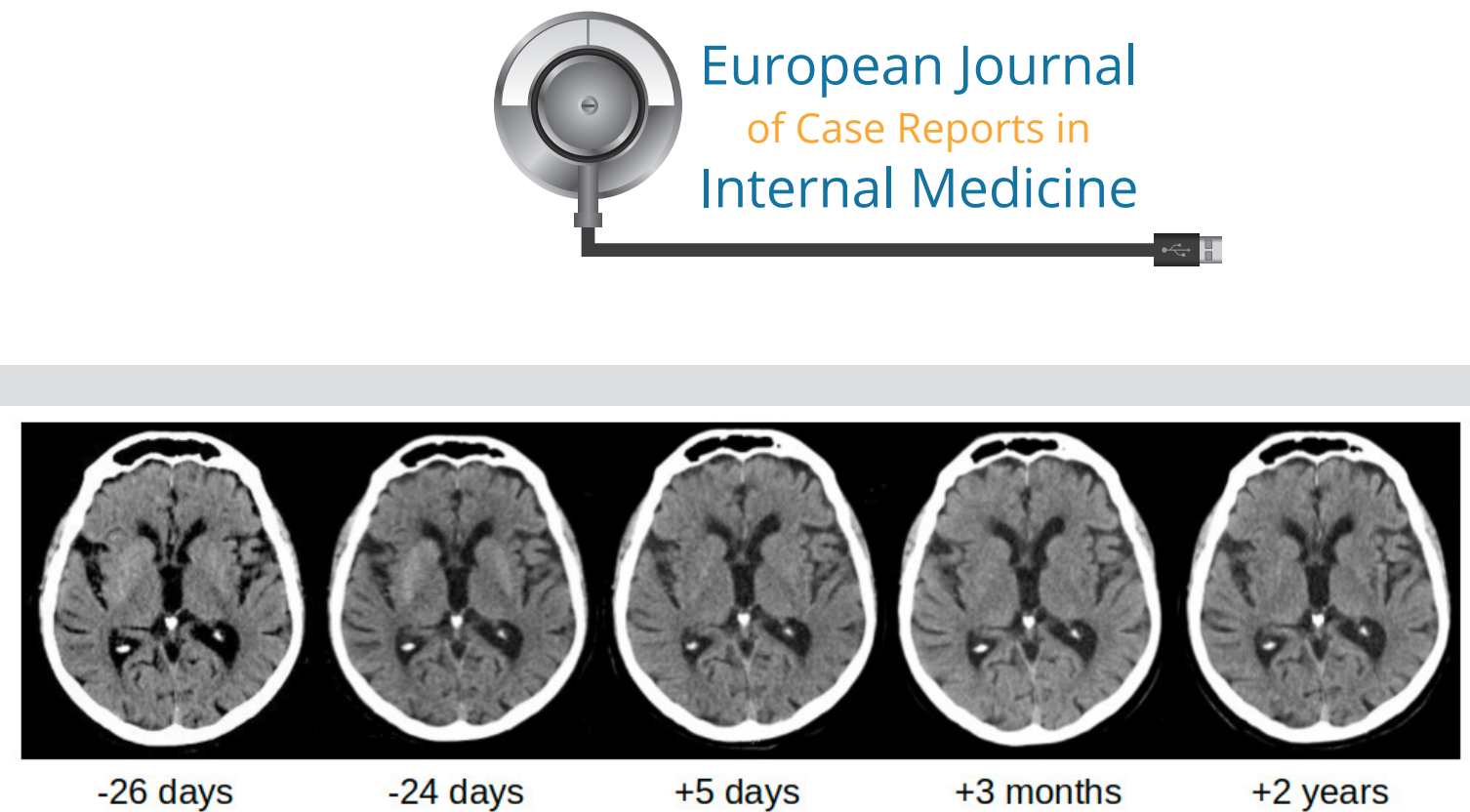

Figure 1. Unenhanced CT scans of the brain obtained with a 40-slice CT scanner Siemens (Germany). Multiplanar reconstruction (MPR), thickness $3 \mathrm{~mm}$ along the bicommissural plane directed through the basal ganglia. Baseline CT scans 26 days and 24 days before hemichorea-hemiballism onset. These images show bilateral striatal hyperdensity before chorea was present. CT scans 3 months later demonstrate resolution of the findings

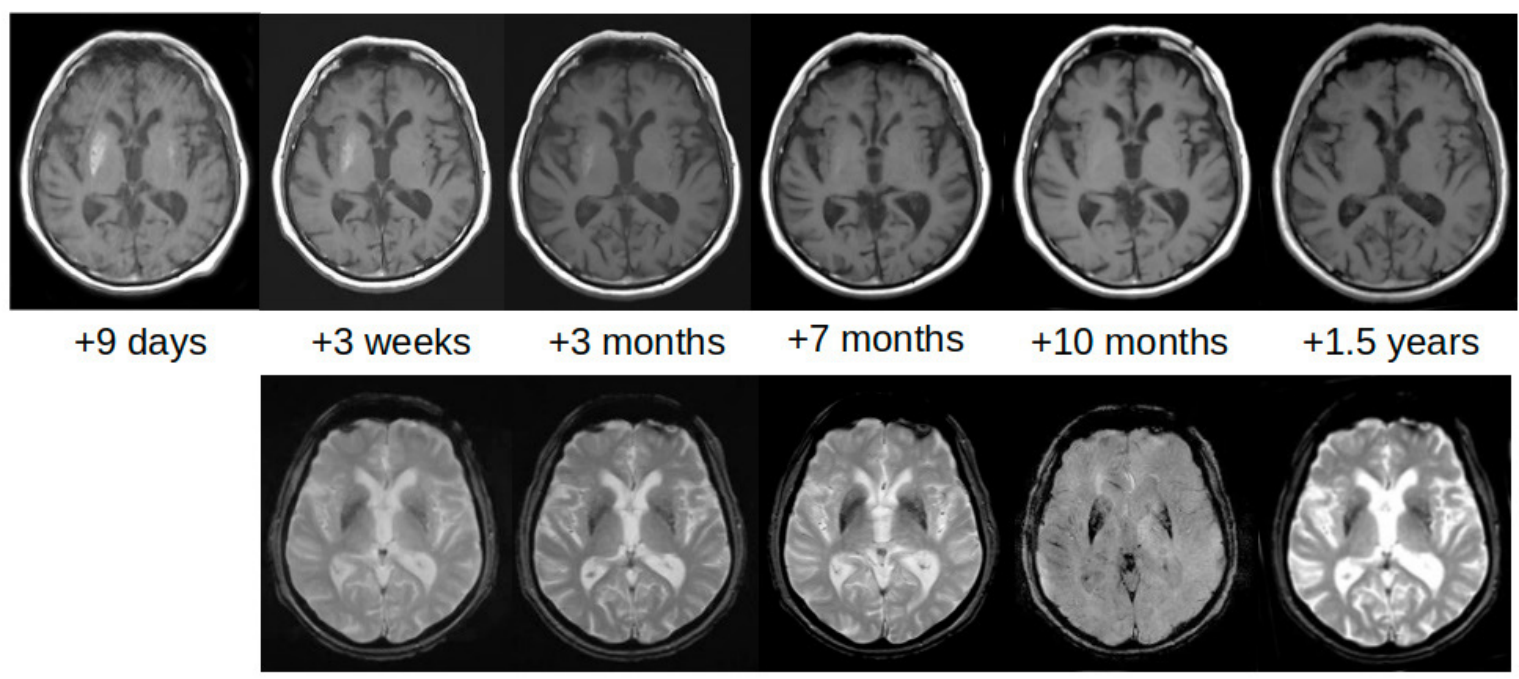

Figure 2. Brain MRI scans performed 9 days after chorea onset show increased T1 signal intensity in both striata on axial T1-weighted imaging, particularly on the right side (upper panel). MRI scans performed 10 months later demonstrate resolution of the findings. However, the same region remained hypointense on susceptibility-weighted imaging (SWI) and gradientecho sequences (T2*-GRE) (lower panel). The MRI scanner was a 1.5T Siemens Avanto (Germany)

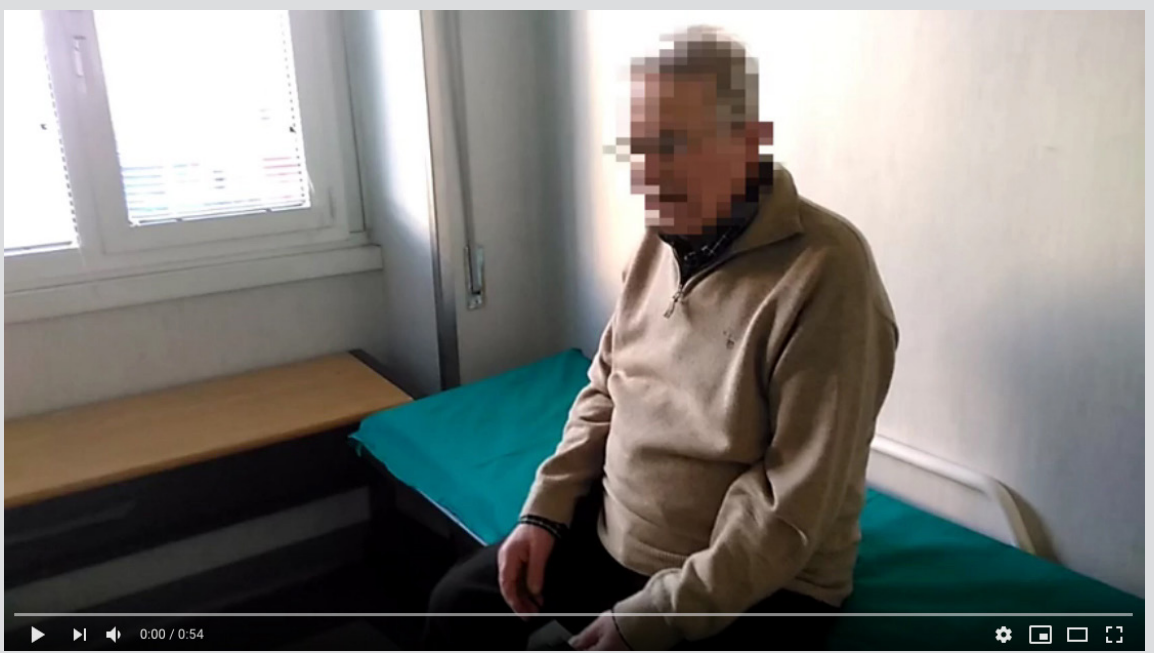

Video 1. Video recorded 3 months after hemichoreahemiballism onset during a follow-up visit. Involuntary movements in the left limbs were greatly reduced but still present, mostly when the patient was speaking. Involuntary movements were mainly choreoathetoid, affecting the distal part of the upper and lower limbs

Watch the video: $h$ ttps://youtu.be/xl4Tipdq2n4 


\section{DISCUSSION}

Our patient underwent repeated brain neuroimaging before $\mathrm{HCHB}$ onset and for a further 2 years. He was admitted because of clinical signs of mild hemiparesis associated with hyperglycaemia and neurological imaging showing subtle bilateral hyperdensity of the striata. Involuntary movements appeared after a mild stroke as for vascular $\mathrm{HCHB}$, but with a glycaemic $\mathrm{HCHB}$ neuroradiological picture. Clinical and neuroradiological findings indicate that our patient had a minor stroke while striatal alterations due to chronic hyperglycaemia were already present but asymptomatic.

Involuntary movements appeared with an unusual delay. While in vascular HCHB the latency between stroke and the appearance of involuntary movement is on average $4.3 \pm 3.6$ (SD) days ${ }^{[10]}$, in glycaemic $\mathrm{HCHB}$ the clinical-neuroradiological timing is unclear. A previous study reported a 1-month delay between hyperglycaemia and hemichorea ${ }^{[11]}$, but no brain neuroimaging was available before the onset of involuntary movements. The only previously described case of glycaemic $\mathrm{HCHB}$ with a brain CT performed before symptom onset indicates that $\mathrm{HCHB}$ may appear as long as 40 days after striatal hyperdensity is detected ${ }^{[9]}$. Our patient already had bilateral asymmetrical striatal CT hyperdensity 26 days before the onset of hemichorea, so our findings confirm that striatal hyperdensity may precede the onset of involuntary movements much earlier than in vascular HCHB.

Our data support the hypothesis that ischaemia could have a role in determining glycaemic $\mathrm{HCHB}^{\left[{ }^{[8}\right.}$. Indeed, there is evidence that hyperglycaemia enhances neuronal necrosis during the acute stage of ischaemic stroke ${ }^{[12]}$. Our patient had multiple vascular risk factors and developed a minor stroke while striatal alterations due to chronic hyperglycaemia were already present on brain CT. A double effect of vascular hypoperfusion and hyperglycaemia could be an unusual mechanism causing neuronal damage in glycaemic $\mathrm{HCHB}$ and its specific neuroradiological picture. Retrospective studies investigating the association between striatal hyperdensity, chronic hyperglycaemia and vascular risk factors could help to elucidate the pathophysiology of glycaemic $\mathrm{HCHB}$.

\begin{tabular}{|c|c|c|c|c|}
\hline $\begin{array}{l}\text { Time with reference } \\
\text { to } \mathrm{HCHB} \text { onset (days) }\end{array}$ & $\begin{array}{l}\text { Blood glucose } \\
\text { (mg/dl) }\end{array}$ & $\begin{array}{c}\text { Glycated Hgb } \\
(\%)\end{array}$ & $\begin{array}{l}\mathrm{CT} / \mathrm{MRI} \\
\text { performed }\end{array}$ & Clinical event \\
\hline-156 & 143 & & & \\
\hline-140 & 186 & & & \\
\hline-26 & 505 & & CT & Mild left hemiparesis \\
\hline-25 & 188 & 15.7 & & \\
\hline-24 & & & CT & \\
\hline-23 & 252 & & & \\
\hline-20 & 236 & 15.1 & & \\
\hline-1 & 112 & & & \\
\hline 0 & & & & $\mathrm{HCHB}$ onset \\
\hline+5 & & & CT & \\
\hline+9 & & & $M R I$ & \\
\hline+21 & & & $M R I$ & \\
\hline+96 & & & $\mathrm{CT}+\mathrm{MRI}$ & \\
\hline+186 & & & $M R I$ & \\
\hline+276 & 114 & 8.7 & & \\
\hline+282 & & & $M R I$ & \\
\hline+515 & & & MRI & \\
\hline+710 & & & CT & \\
\hline
\end{tabular}

Table 1. Timing and results of blood glucose and glycated haemoglobin tests and brain neuroimaging HCHB, hemichorea-hemiballism. 


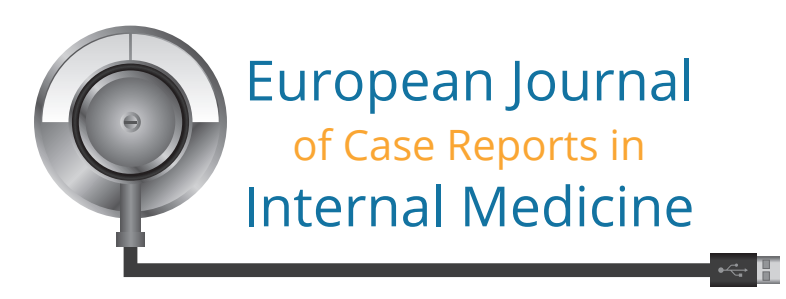

\section{REFERENCES}

1. Piccolo I, Defanti CA, Soliveri P, Volontè MA, Cislaghi G, Girotti F. Cause and course in a series of patients with sporadic chorea. J Neurol 2003;250(4):429-435.

2. Cosentino C, Torres L, Nuñez Y, Suarez R, Velez M, Flores M. Hemichorea/hemiballism associated with hyperglycemia: report of 20 cases. Tremor Other Hyperkinet Mov (N Y) 2016;6:402

3. Tocco P, Barbieri F, Bonetti B, Barillari M, Marangi A, Tinazzi M. Hemichorea-hemiballismus in patients with non-ketotic hyperglycemia. Neurol Sci 2016;37(2):297-298.

4. Shafran I, Greenberg G, Grossman E, Leibowitz A. Diabetic striatopathy-Does it exist in non-Asian subjects? Eur J Intern Med 2016;35:51-54.

5. Yu F, Steven A, Birnbaum L, Altmeyer W. T2*-based MR imaging of hyperglycemia-induced hemichorea-hemiballism. J Neuroradiol 2017;44(1):24-30.

6. Goh LW, Chinchure D, Lim TC. Clinics in diagnostic imaging (166). Nonketotic hyperglycaemic chorea-hemiballismus. Singapore Med J $2016 ; 57(3): 161-164$; quiz 165.

7. Hashimoto T, Hanyu N, Yahikozawa H, Yanagisawa N. Persistent hemiballism with striatal hyperintensity on T1-weighted MRI in a diabetic patient: a 6-year follow-up study. J Neurol Sci 1999;165(2):178-181.

8. Shan D-E. Hemichorea-hemiballism associated with hyperintense putamen on T1-weighted MR images: an update and a hypothesis. Acta Neurol Taiwan 2004:13(4):170-177.

9. Nakajima N, Ueda M, Nagayama H, Katayama Y. Putaminal changes before the onset of clinical symptoms in diabetic hemichorea-hemiballism. Intern Med 2014;53(5):489-491.

10. Alarcón F, Zijlmans JCM, Dueñas G, Cevallos N. Post-stroke movement disorders: report of 56 patients. J Neurol Neurosurg Psychiatry 2004;75(11):1568-1574.

11. Lin C-J, Huang P. Delayed onset diabetic striatopathy: hemichorea-hemiballism one month after a hyperglycemic episode. Am J Emerg Med 2017;35(7):1036.e3-1036.e4.

12. Sulter G, Elting JW, De Keyser J. Increased serum neuron specific enolase concentrations in patients with hyperglycemic cortical ischemic stroke. Neurosci Lett 1998;253(1):7173. 